\title{
Excitation of L-valine molecules by electrons and photons
}

\author{
Yu. A. Bandurin ${ }^{1, a}$, A. N. Zavilopulo ${ }^{1, b}$ (D) Sh. Molnar ${ }^{2}$, and O. O. Shpenik ${ }^{2}$ \\ 1 Institute of Electron Physics, National Academy of Sciences of Ukraine, Uzhhorod 88017, Ukraine \\ ${ }^{2}$ Ukrainian-Hungarian Educational-Scientific Institute of Uzhhorod National University, Uzhhorod 88000, Ukraine
}

Received 30 August 2021 / Accepted 15 December 2021 / Published online 17 January 2022 (C) The Author(s), under exclusive licence to EDP Sciences, SIF and Springer-Verlag GmbH Germany, part of Springer Nature 2022

\begin{abstract}
Excitation of L-valine molecules was studied by optical spectroscopy. Optical emission spectra of the L-valine molecule and optical excitation functions of molecular bands and the $\mathrm{H}_{\beta}$ spectral line were measured in the gas phase using electron impact. In the spectra of optical emission in the wavelength range of $250-500 \mathrm{~nm}$, intense emission bands were found at energies of incident electrons of 30,50 and $70 \mathrm{eV}$. Analysis of structural features of the valine molecule suggested a fragmentation scheme with the formation of excited particles in collisions with electrons. A notable feature of the presented optical excitation functions is a different growth dynamics with an increase in the energy of exciting electrons and the presence of a number of features and kinks, which are especially pronounced for $\lambda=305 \mathrm{~nm}$ and $\lambda$ $=311 \mathrm{~nm}$. The excitation thresholds were determined from the initial sections of the excitation functions of the most intense spectral lines by the least-squares method. The photoluminescence spectra of L-valine were measured for the first time on a Shimadzu RF-6000 spectrofluorophotometer in the spectral range of 400-800 $\mathrm{nm}$ for excitation wavelengths of 250, 275, 333, 351, and $380 \mathrm{~nm}$.
\end{abstract}

\section{Introduction}

Excitation of atoms, ions and molecules by electron impact is a key elementary process that determines the basic properties of matter in the gas and plasma phases. It is the process of particle excitation in collisions with electrons that determine the features of photon emission in the form of molecular bands and spectral lines. Excitation of molecules into repulsive states leads to dissociation, which determines the concentration of various atomic particles. In addition, collisions of electrons with molecules are accompanied by vibrational and rotational excitations of molecular energy levels. Similar processes occur when molecules are excited by photons, and photodissociation is the main accompanying process. The energies of photons of visible and ultraviolet radiation are sufficient both to break a chemical bond and to change the structure of an excited molecule. In this case, the processes of molecular excitation are accompanied by transitions of one or more electrons to higher electronic states, followed by emission in a wide range of wavelengths from infrared to ultraviolet. Investigation of the processes of excitation by electron and photon impact can be carried out by different methods, the most effective one is the optical method.
Processes occurring in polyatomic organic molecules at their interaction with slow electrons are of considerable interest not only for quantum theory of scattering and theory of electronic structure of molecules but also for solving a number of applied problems [1-3]. One of the most important elementary processes occurring in molecules when they interact with slow electrons is the process of excitation, which leads to the emission of spectral bands by both the whole molecules and their fragments. The study of molecules in the gas phase makes it possible to exclude solvation effects that can affect conformational stability of molecules and redistribution of vibrational excitations in real biological systems. Among the elementary processes occurring at the interaction of low-energy electrons with molecules, the processes of elastic scattering, excitation and ionization are the most effective $[4,5]$.

Studies of various processes of interaction of lowenergy (slow) electrons with molecules that are part of DNA and RNA are of particular interest. These processes are a subject of intensive research [6-10]. It should be noted that secondary electron emission, which is the result of the primary interaction of electrons with a molecule, plays an important role in the mechanism of radiation damage to DNA. The destructive role of these secondary electrons is the further ionization of the peptide components of DNA, initiating an avalanche effect, leading to damage to the genome.

\footnotetext{
${ }^{a}$ e-mail: bandurinunc@ukr.net

b e-mail: gzavil@gmail.com (corresponding author)
} 
Therefore, first of all, attention is paid to the elucidation of mechanisms of processes that damage the structure of these molecules, which lead to the death of individual cells and the organism as a whole. The problem with the basic components of DNA and RNA often requires solutions, since an accurate understanding of the processes occurring at the molecular level is considered an important step towards describing more complex phenomena occurring at the cellular level. According to the prevailing concepts [11], it is slow electrons that are related to the main part of destructive changes at the molecular level of biostructures, with the main target being genetic macromolecules [12]. The latter is important, in particular, for studying mechanisms of mutations in biological objects during a viral pandemic. The relevance of studying these processes by various methods, especially in the case of biomolecules, has acquired particular importance at the present time in relation to the COVID-19 pandemic [13, 14].

The range of low energies of the exciting electrons includes characteristic values of the binding energies of atoms in organic molecules, excitation energies, and ionization potentials of atoms and molecules themselves, which makes it possible to study the elementary processes of excitation, ionization, and dissociation. Consequently, information about processes at atomic and molecular levels can be considered the first stage in the study of the general mechanisms of functioning of a living cell. An applied aspect of this problem is the possibility of a directed change in the parameters of such biostructures, which will lead to the creation of new bioorganic materials.

Application of monoenergetic electron beams with scanning of their energy to excite complex molecules in the gas phase enables one to obtain information on the position and structure of energy levels, as well as to estimate the relative probability of their excitation. Investigation of emission of photons and energy dependences of the excitation of electronic-vibrational states provides information on the efficiency of conversion of internal energy of complex molecules into the light.

Despite a definite importance of studying the main mechanisms of structural changes in amino acid molecules under the action of low-energy electrons, there are few data of this kind and they belong to two areas of research. The first one is related to mass spectrometric experiments, in which electrons with an energy of $70-100 \mathrm{eV}$ are used to ionize bioorganic molecules. Among such works on the determination of the mass spectra of molecules that make up amino acids should be mentioned: thymine [15], adenine [16-18], cytosine [18], uracil [19], guanine [20], valine [21], and glutamine and glutamic acid [22]. Another line of research is the study of elementary processes of excitation, dissociation and ionization of such molecules under the impact of electrons and photons [22-26].

We also point out the importance of processes with the formation of molecular negative ions (FNI), in the energy range $0-5 \mathrm{eV}$. Their formation occurs according to resonance mechanisms and is described in terms of the energies and symmetry of vacant molecular orbitals.
The decay of negative ions is possible or by the auto detachment of an electron, or dissociation into fragments. It is determined by the fundamental characteristics of the target molecule and depends on the number of vibrational degrees of freedom, spatial structure, vibrational state, and electron affinity. A number of interesting works [22-26] are devoted to these processes; the most complete review of the current state and prospects of the spectroscopy of dissociative electron capture was performed in [22]. For biomolecules, for example, in [23], total dissociative electron capture cross-sections for thymine, cytosine, and adenine and for three compounds used as surrogates for the ribose were investigated. It was shown that cross-sections are roughly comparable magnitudes.

It was shown in [24] that slow electrons (less than $3 \mathrm{eV}$ ) effectively decompose uracil (U) in the gas phase, forming the (U-H) anion, which is explained by the high electron affinity to the (U-H) radical. This process has important implications for the radiation damage. In [25-27], the formation of negative ions of cytosine and thymine molecules was investigated in the process of dissociative ionization and their total cross-sections were measured. Another line of research is the study of elementary processes of excitation, dissociation and ionization of such molecules by electrons and photons impact [28-33].

This paper presents the results of the first experiments to study the optical spectra of valine molecules excited by electron impact. Valine is important as a radioprotector in radiation therapy $[27,28]$, and it also takes part in the synthesis of nucleic acids and enzymes that carry out energy redox reactions at the cellular level. We investigated the emission spectra of L-valine molecules in the gas phase upon excitation by lowenergy electrons, measured the optical excitation functions (OEF) of individual emission bands, and identified the mechanisms of their excitation. An experimental study of the photoluminescence spectra of L-valine in the solid phase under excitation by photons is also performed.

\section{Features of the structure of the molecule of valine}

When electrons collide with molecules, electronic $\left(E_{\mathrm{e}}\right)$, vibrational $\left(E_{\mathrm{k}}\right)$ and rotational $\left(E_{\mathrm{v}}\right)$ levels of molecules can be excited, in accordance with the relationship $E_{\mathrm{e}}$ i. $E_{\mathrm{k}} i E_{\mathrm{v}}$.

It should be noted that the excitation of molecules by electron impact in the energy range from 0 to $20 \mathrm{eV}$, where are located the lower singlet and triplet excited levels and ionization potentials of the parent and daughter components of the molecule, can lead in some cases to dissociative excitation. In this case, several components of the molecule are simultaneously excited due to the internal redistribution of the energy of the incident electrons. 
Dissociation occurs as a result of the concentration of vibrational energy on the weakest bond. In polyatomic molecules and radicals, a change in the rotational structure in the emission spectrum is possible as a result of predissociation due to a nonradiative transition of an excited molecule from a stable electronic state to an unstable one with the same energy at which dissociation occurs. For complex molecules, predissociation is very difficult to observe because of numerous vibrational degrees of freedom. However, it is precisely for such molecules that the role of predissociation in their decay upon excitation of energy levels lying above the dissociation limits is important.

In polyatomic molecules and radicals, the identification of the rotational structure in the emission spectrum due to predissociation is very difficult. From the break in the molecular structure, it is impossible to conclude that the predissociation limit allows one to determine the dissociation limit of excitation. However, the lower predissociated level gives an exact upper limit for one of the dissociation energies of the HNO radical i $2.14 \mathrm{eV}$ [34].

Amino acids are biologically important organic compounds that are the building blocks of proteins and contain amine $\left(-\mathrm{NH}_{2}\right)$ and carboxyl $(-\mathrm{COOH})$ groups. Amino acids, after water, are the most abundant components of tissue cells in living organisms. Most proteins consist of a combination of nineteen so-called "primary" amino acids, that is, containing a primary amino group, and one "secondary" amino acid proline or imino acid (contains a secondary amino group), which are called standard or proteinogenic amino acids [5]. Amino acid molecules exist in various conformers. The carboxyl group can rotate, and the hydrogen atom can be oriented both in the direction of nitrogen and in the opposite direction. Depending on which carbon atom of the molecule the amino group is attached to, amino acids are divided into $\alpha-, \beta$-, $\gamma$-, etc., the $\alpha$-atom is the carbon atom connected to the carboxyl group. In addition, the conformational variability of molecules contributes to the reorientation of the flexible - $\mathrm{COOH}$ and amine $-\mathrm{NH}_{2}$ groups, forming various intramolecular hydrogen bonds, and their residue, called the R-group or side chain, may also contain other elements. For example, it's possible to link an unseparated pair of nitrogen atoms with the hydrogen of the hydroxyl group ( $\mathrm{N}$... $\mathrm{HO}$ ), or establish a bond between the hydrogen atom of the amine group and the oxygen atom of the carbonyl $(\mathrm{NH} \ldots \mathrm{O}=\mathrm{C})$ and hydroxyl $(\mathrm{NH} \ldots \mathrm{OH})$ groups.

It is obvious that the interaction of hydrogen bonds plays an important role in the conformational energy and frequencies of harmonic vibrations. It should be noted that the structure of isolated valine molecules has not been experimentally established; apparently, this is due to the high conformational lability of valine, which has four intramolecular rotational degrees of freedom. There are a number of theoretical works on the calculation of the structure of the conformers of the valine molecule. In [30, 31], a method was developed for determining the structure (conformational composition) of molecules in an isolated state, which included a direct comparison of the experimental method of low-temperature matrix IR spectroscopy with quantum mechanical calculations. This made it possible, based on the analysis of multidimensional potential energy surfaces, to determine all possible conformational states. Work [32] is devoted to the study of the conformational properties and vibrational spectra of the L-enantiomeric structures Val, Leu, and Ile. For the valine molecule, the energies for the 12 lowest-energy conformers were calculated. It has been shown that the relative stability of such conformers depends mainly on the interaction of the $\mathrm{H}$-bond and the orientation of the side chain. The strength of a hydrogen bond depends on the type of hydrogen bonding, as well as the torsion of $\mathrm{NH}_{2}$ and the $\mathrm{COOH}$ group.

Figure 1 shows the structural 3D, lines and Dvaline, and L-valine formulas of the valine molecule $\mathrm{C}_{5} \mathrm{H}_{11} \mathrm{NO}_{2}$, as well as the structure of the most stable neutral conformations of L-valine molecules. Note that the asymmetric carbon atom $(\mathrm{C} \alpha$-atom $)$ in $\alpha$-amino acids leads to the appearance of optical isomers (mirror antipodes), which play an important role in the processes of protein biosynthesis. In addition, aliphatic amino acids can be considered as model systems that imitate the process of interaction of ionizing radiation with large biomolecular complexes (proteins, peptides). It is known [11] that when exposed to ionizing radiation, secondary particles arise, such as radicals or electrons with energies below $20 \mathrm{eV}$, which play an important role in the generation of defects in a biological system. The energy of secondary electrons is sufficient to cause excitation, dissociation, electron capture and/or electron ionization of the molecules, initiating additional structural damage.

As follows from Fig. 1 in the structure of the valine molecule a carbon atom is bonded to 4 substituents, one of them is a hydrogen atom, the second one is the carboxyl group $\mathrm{COOH}$, the third one is the amino group $\mathrm{NH}_{2}$ capable of attaching a hydrogen ion, the fourth one is an alkyl side chain of $\mathrm{C} \alpha$ atoms, the composition of which determines the basic properties of this amino acid. The enantiomer L-valine should have the same energy characteristics: heats of formation, excitation and ionization potentials, activation energy [11].

Elucidation of physical principles of formation of the unique structure of biomacromolecules and mechanisms of their functioning has been considered an urgent problem in molecular biophysics for decades. It is so natural to count the pictures of symmetry and violations of symmetry by a fundamental factor in consideration of physical bases of the structure of the living systems, their origin and evolution. Thermodynamics disequilibrium, non-linearity, hierarchy, synergism, combination of phases, conjugation of quantum, and macroscopic structures and processes, show up, for example, as a cellular structure of all living, as an exchange of matters, as excitability, as enzymatic catalysis, as vertical division of managing and performance functions. Chirality is the property of a molecule not to be combined in space with the mirror image [35]. Figure 1b illustrates the chirality of a valine molecule. Chiral duality 

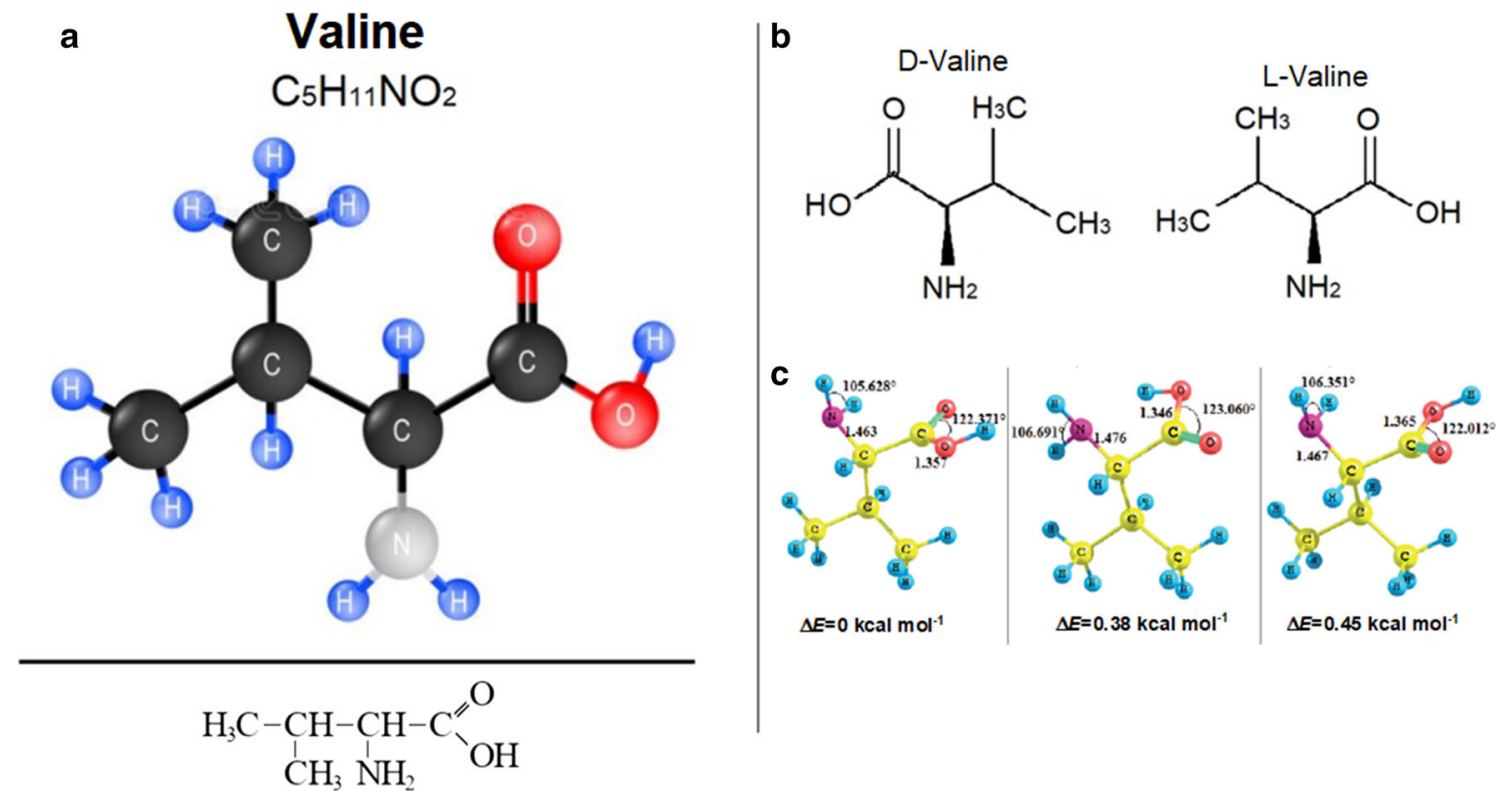

Fig. 1 Structural 3D and lines formulas of the valine molecule a; the difference between the structure of D-valine and Lvaline $\mathbf{b}$; structure of the most stable neutral conformations of L-valine molecules and comparison of the molecular energy relative to the conformation, the energy of which is $-402.44 \mathrm{kcal} / \mathrm{mol}$ is taken as 0 c [31]

becomes the natural tool for combinatorics of structural correlations in these subsystems at all levels of structural organization. The phenomenon of chiral dualism can be examined as a unique physical tool of molecular biology, related to a phenomenon of symmetry violation and enabling hierarchical structures of proteins and nucleic acids to be formed. Proteins are composed of L-forms of optically active amino acids. There is no unambiguous connection between the configuration of the structure and the sign of rotation: similar structures with the same sign of rotation can have opposite configurations. We began studying L-valine photon emission, hoping afterward to find out the influence of other molecular structural forms of both at an electron impact and at the study of luminescence.

\section{Experimental setups}

\subsection{Installation for excitation by electrons}

The block diagram of the experiment is shown in Fig. 2. The measurements were carried out by the optical method using a vapor-filled cell (3), in which the required concentration of valine molecules was created in the zone of their interaction with electrons [36, 37]. The container with the studied molecules (2) was located in close proximity $(\sim 3 \mathrm{~cm})$ to the cell and had independent heating. The container temperature was maintained in the range of $123-126{ }^{\circ} \mathrm{C}$. To prevent the condensation of vapor on the structural elements of the electron gun (1), their temperature was by $20-30^{\circ} \mathrm{C}$ higher than the container temperature. A heater was also installed in front of the output quartz window on the side surface in the vacuum part of the chamber to prevent film deposition on the window surface. The residual gas pressure in the vacuum chamber under operating conditions did not exceed $1.3 \cdot 10^{-4} \mathrm{~Pa}$.

A collimated electron beam with a diameter of $2 \mathrm{~mm}$ and a current of $\sim 10 \mu \mathrm{A}$ in the energy range of $1-120 \mathrm{eV}$ crosses the vapor-filled cell and is detected by a Faraday cup. The instability of the current of electrons passing through the collision chamber did not exceed 3\%. The monoenergetic of the electron beam (full width at half maximum) was not worse than $0.25 \mathrm{eV}$ (see Fig. 2a) and was determined automatically before each measurement by differentiating the current-voltage (I-V) characteristic. The energy of electrons during the measurements of optical excitation functions in a given energy range was scanned by a measurement system (2) with a step in the range of 100-400 meV.

Photon emission analyzed by an MDR-2 diffraction monochromator (6) was detected by a photomultiplier tube (7). The system for automatic control of parameters and registration of the useful signal (5) made it possible to pre-amplify and shape single-photon electronic pulses from the photomultiplier with a broadband discriminator amplifier and send them for processing and recording to a personal computer. Accumulation of the useful signal to ensure the required measurement accuracy was continued until the statistical spread in the number of pulses did not exceed 100-200 impulses/s, and the exposure at each point was within $10 \div 40 \mathrm{~s}$.

Optical emission spectra in the wavelength range $\lambda$ $=250-500 \mathrm{~nm}$ were measured with a step of $0.814 \mathrm{~nm}$, and the entrance slit of the monochromator of $1 \mathrm{~mm}$ provided the transmission of the spectral interval $\lambda=$ 


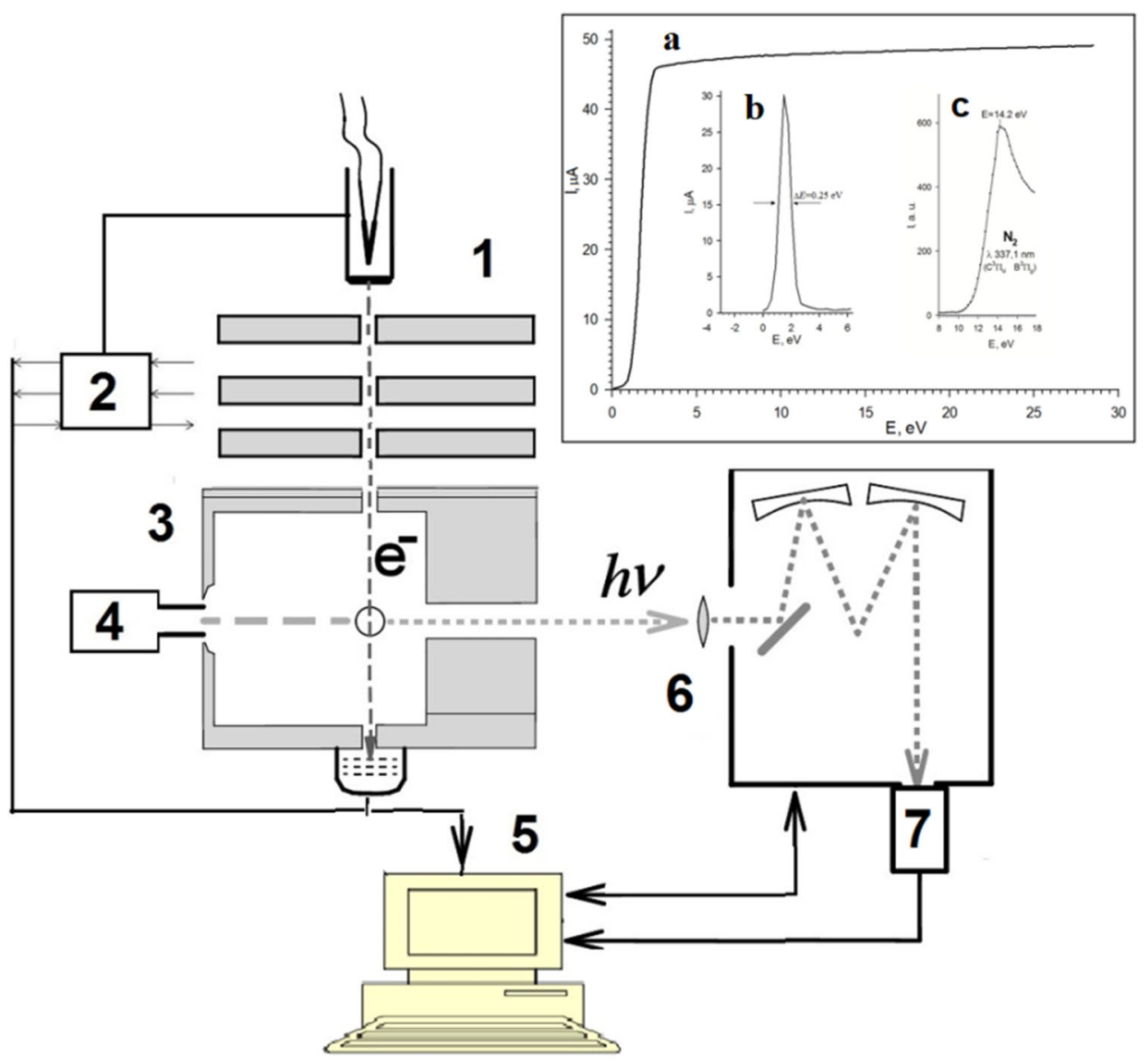

Fig. 2 The basic block diagram of the experimental setup: 1-cathode and electrodes of the electron gun; 2-modular power supply and control units; $\mathbf{3}$-vapor-filled cell; $\mathbf{4}$-container with the investigated molecules; 5 - system for automatic control of parameters, registration of the useful signal and control of the experiment; 6-MDR-2 monochromator; 7-Photomultiplier tube with power supply. Insert: $\mathbf{a}-\mathrm{I}-\mathrm{V}$ characteristics; $\mathbf{b}$ - function of the electron energy distribution obtained by differentiating the initial section of the I-V characteristic of the electron gun; c- the initial section of the excitation function of the spectral band of the nitrogen molecule $\lambda=337.1 \mathrm{~nm}\left(\mathrm{C}^{3} \Pi_{\mathrm{u}} \rightarrow \mathrm{B}^{3} \Pi_{\mathrm{g}}\right)$ used for calibration

$2 \mathrm{~nm}$. To exclude the influence of the background illumination on the value of the useful signal, the electron beam was modulated and subtracted from the total signal.

An important factor for correct analysis of the measurement data is the calibration of the energy scale of the exciting electron beam. We calibrated it by two methods: (1) by the shift of the $\mathrm{I}-\mathrm{V}$ characteristic of the electron current to the collector, and (2) by the known optical excitation function (OEF) of the spectral lines of the mercury atom and nitrogen molecule. The excitation threshold of the $\mathrm{Hg}$ I $253.7 \mathrm{~nm}$ resonance line is $4.885 \mathrm{eV}$, which made it possible to fix the initial point of the energy scale. The second point was determined from the position of a sharp maximum of the OEF of the spectral band of the second positive system of the nitrogen molecule $\lambda=337.1 \mathrm{~nm}$ $\left(\mathrm{C}^{3} \mathrm{P}_{\mathrm{u}} \rightarrow \mathrm{B}^{3} \mathrm{P}_{\mathrm{g}}\right)$. The difference between the energy position of the indicated maximum $(E=14.2 \mathrm{eV})$ in the excitation cross-section of the nitrogen molecule with what we measured made it possible to determine the contact potential difference, which coincided with an accuracy of $\pm 50 \mathrm{meV}$, with the value determined by the methods indicated above. Thus, the relative uncertainty of the electron energy scale which we determined when measuring the OEF was not worse than $\pm 0.1 \mathrm{eV}$.

\subsection{Setup for excitation by photons}

To study the luminescence spectra of L-valine powder (Sigma Aldrich, assay $\geq 99.5 \%$ (NT)), tablets $25 \mathrm{~mm}$ in diameter and 1-mm thick were prepared by pressing. Figure 3 shows a schematic diagram of an experiment to study the processes of excitation by photons. A xenon lamp or a laser were used as a source of photons (1), the radiation passed through a UV filter (2) and was directed to a sample located on a special holder of the device (3) strictly vertically. Luminescence radiation through a filter (4) was directed to the entrance slit of the monochromator (5) and recorded by a detector $(\mathbf{6})$. The signal from the detector was processed and sent to the A/D conversion (7), and a USB-port of a 


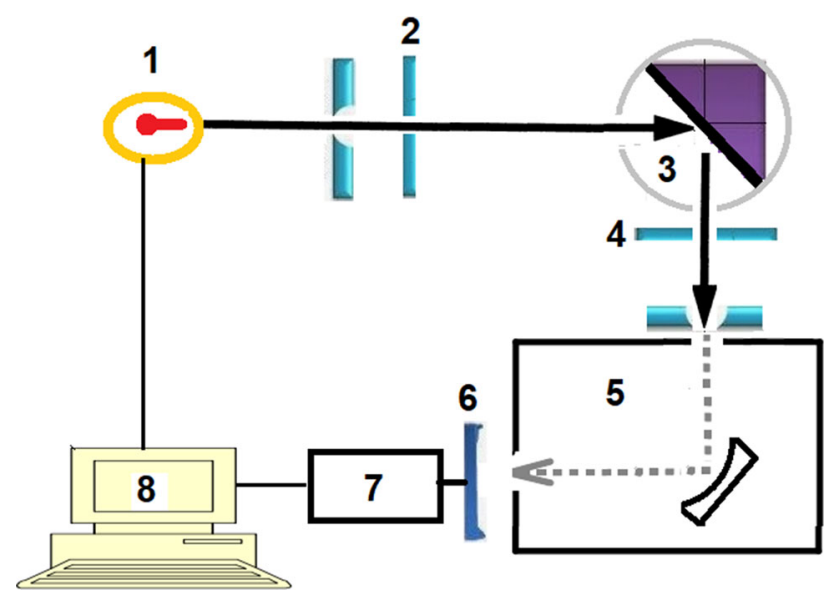

Fig. 3 Schematic of an experiment to study the processes of excitation by photons: $\mathbf{1}$ - excitation light, $\mathbf{2}$-UV filter, 3-sample holder, 4 - excitation filter, 5-monochromator, 6- detector, 7-A/D conversion circuit, 8-computer

computer (8). The experiment was controlled automatically by a personal computer. Measurements, control, processing of the useful signal is carried out automatically using the intuitive LabSolutions RF software and allows you to work in the following modes [38]:

- spectral-registration of excitation and emission spectra with the possibility of subsequent processing of spectra (determination of the position of maxima and minima, determination of the measured value at selected points, calculation of the area, subtraction of spectra, derivatives of 1-4 orders, smoothing, arithmetic operations and logarithmization)

- 3D scanning mode (high speed): registration of excitation, emission and synchronization spectra, presentation of the obtained data in the form of a 3-D spectrum

- quantitative-building a calibration dependence and calculating an equation of 1-3 orders of magnitude, averaging measurements, determining the concentration of impurities in unknown samples

- photometric - measurement of fluorescence intensity at one or more wavelengths

- time - registration of signal changes in time.

The RF-6000 Photofluophotometer has a high sensitivity and a wide dynamic range, which makes it possible to measure the spectra of fluorescence, bioluminescence, chemiluminescence, and electroluminescence of various samples: liquid or solid. The combination of an automatic mode of searching for the optimal excitation/emission wavelength of the luminescence and a high scanning speed enables fast measurements and prevents photo destruction of samples, which is very important for studies of biological molecules.

Light from the xenon lamp 1 through a U-340 filter $(\mathbf{2})$ (transmission band $200 \div 400 \mathrm{~nm}$, coefficient of transmittance $-45 \%$ at the wavelength $275 \mathrm{~nm}$ ) falls on the surface of L-valine (3). Before the entrance slit
Table 1 Binding energies and excitation wavelength range

\begin{tabular}{llll}
\hline Bond & $\begin{array}{l}\text { Binding } \\
\text { energy, } \\
\text { eV }\end{array}$ & $\begin{array}{l}\text { Range of } \\
\text { excitation } \\
\text { wavelength, nm }\end{array}$ & $\begin{array}{l}\text { Photon energy } \\
\text { interval, eV }\end{array}$ \\
\hline H-O & - & $247.5 \div 252.5$ & $4.91 \div 5.01$ \\
H-O & 4.52 & $272.5 \div 277.5$ & $4.47 \div 4.55$ \\
H-C & 4.2 & $300.5 \div 335.5$ & $3.7 \div 3.75$ \\
H-N & 3.83 & $348.5 \div 353.5$ & $3.51 \div 3.56$ \\
C-C & 3.61 & $377.5 \div 382.5$ & $3.242 \div 3.284$ \\
C-O & 3.31 & - & - \\
C-N & 2.89 & & \\
N-O & 1.7 & & \\
\hline & & &
\end{tabular}

of the detection system we put an L- 42 optical filter 4 (transmission $\geq 400 \mathrm{~nm}$, coefficient of transmittance more than $92 \%$ ). At the initial stage, the experiment to study luminescence was carried out by irradiating the sample at an angle of $45^{\circ}$ to the surface (see Fig. 3). It turned out that the number of reflected and scattered photons in some cases exceeds the useful signal from the luminescence. To change the angle of incidence of photons (to reduce the number of reflected and scattered photons), a special sample holder was made that allows one to change the angle of incidence and determine the optimal angle of incidence for the signal/background ratio. The results presented here were performed at an angle of incidence of exciting photons at the surface equal to $15^{\circ}$ and the observation angle of the luminescence radiation of $75^{\circ}$.

The experimental technique consisted of the excitation of luminescence by five different wavelengths. Before the start of luminescence measurements, a background signal from the black surface was recorded for each exciting light wavelength, which was subsequently subtracted. When measuring the background, a black plate was placed in the place of the valine sample. Initially, at an exciting light wavelength $\lambda=275 \pm 2.5 \mathrm{~nm}$ (photon energy $E=4.52 \mathrm{eV}$ ), which corresponds to the binding energy of free an $\mathrm{OH}$ radical, the luminescence spectrum was measured. Having determined the position of the maximum $\lambda=503 \mathrm{~nm}$, the excitation spectra in the short wavelength region of $200-400 \mathrm{~nm}$ was measured at this wavelength. This spectrum shows several peculiarities at different wavelengths.

We used five excitation wavelengths (see Table 1) with the photon energies corresponding to the binding energies of different diatomic molecules. One of the wavelengths $\lambda=250 \pm 2.5 \mathrm{~nm}$ corresponds to the photon energy higher than any binding energy in the L-valine molecule. We did not use the wavelength for the excitation of the $\mathrm{N}-\mathrm{O}$ bond $(1.7 \mathrm{eV})$ because $\lambda=$ $729 \mathrm{~nm}$ is out of the excitation range of $200 \div 400 \mathrm{~nm}$. 


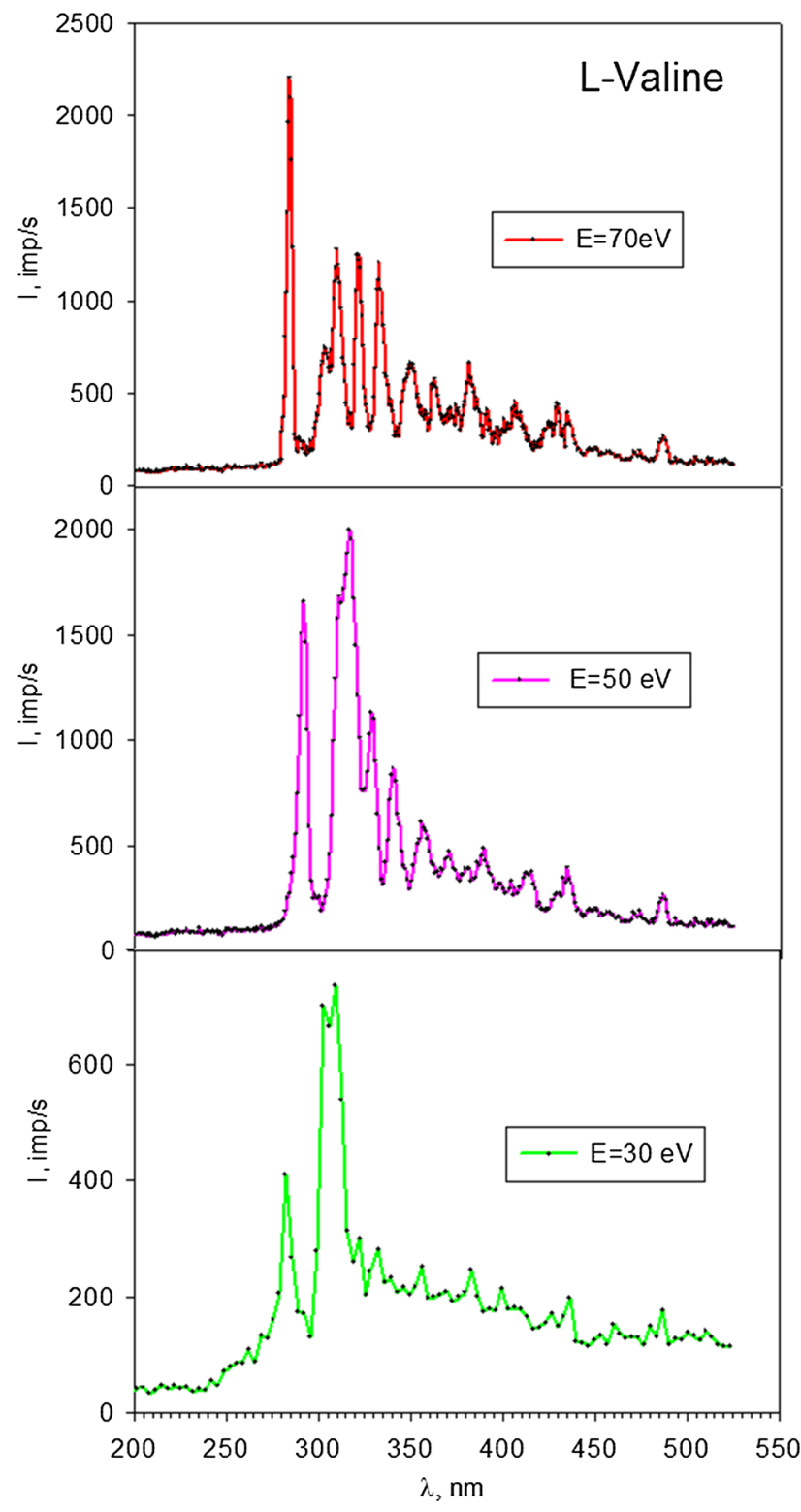

Fig. 4 Optical emission spectra of the L-valine molecule by electron impact

\section{Results and discussion}

\subsection{Optical spectra under electron impact}

We measured the emission spectra during the bombardment of valine molecules by electrons in the energy range $30-70 \mathrm{eV}[36,39]$. The measurement procedure included two stages: at the first stage, the emission spectra of valine molecules at different electron energies were recorded, and at the second stage, the OEF of the most intense molecular bands and atomic spectral lines were measured.

Figure 4 shows optical emission spectra of L-valine molecule at the bombarding electrons energies of 30 , 50 , and $70 \mathrm{eV}$. As can be seen, the spectra obtained depend significantly on the energy of the bombarding electrons. While at an electron energy of $30 \mathrm{eV}$ only one intense emission band at $300-320 \mathrm{~nm}$ and a weak one at 270-290 $\mathrm{nm}$ are revealed at electron energies of $50 \mathrm{eV}$ and $70 \mathrm{eV}$, a number of separate narrow bands additionally in the long-wavelength region is observed.

In the investigated wavelength range, presumably at the given electron energies, there can be spectral lines and molecular bands emitted by excited neutral fragments of the valine molecule - atomic $\mathrm{H}, \mathrm{C}, \mathrm{N}, \mathrm{O}$, molecular diatomic $\mathrm{H}_{2}, \mathrm{OH}, \mathrm{CO}$, and polyatomic $\mathrm{NH}_{2}$, $\mathrm{CH}_{3}, \mathrm{COOH}$ (see the structure in Fig. 1). It can also be expected that at electron energies up to $100 \mathrm{eV}$ in the gas phase of molecules, the formation of excited positive molecular fragment-ions is possible. As a rule, the emission of photons by excited $\mathrm{C}, \mathrm{N}, \mathrm{O}$ atoms is by several orders of magnitude less efficient than the emission of $\mathrm{H}$ atoms. Similarly, the emission of molecular bands by excited $\mathrm{NH}_{2}, \mathrm{CH}_{3}$, $\mathrm{COOH}$ fragments is insignificant in comparison with $\mathrm{OH}$ and $\mathrm{CO}$ molecules.

Identification of emitters of molecular bands is difficult both due to the formation of different fragments of the valine molecule due to electron impact and due to the coincidence of the emission wavelengths of different emitters. Analyzing the structural diagrams of the valine molecule (see Fig. 1), one can be assumed that its fragmentation with the formation of excited particles in collisions with electrons can occur according to the following schemes:

$$
\mathrm{C}_{5} \mathrm{H}_{11} \mathrm{NO}_{2}+\mathrm{e}^{-} \rightarrow \begin{cases}\mathrm{C}_{5} \mathrm{H}_{10} \mathrm{NO}_{2}+\mathrm{H}^{*}+\mathrm{e}^{-} & \text {(a) } \\ \mathrm{C}_{5} \mathrm{H}_{10} \mathrm{NO}+\mathrm{OH}^{*}+\mathrm{e}^{-} & \text {(b) } \\ \mathrm{C}_{4} \mathrm{H}_{10} \mathrm{~N}+\mathrm{OH}^{*}+\mathrm{CO}^{*}+\mathrm{e}^{-} & \text {(c) } \\ \mathrm{C}_{4} \mathrm{H}_{10} \mathrm{~N}+\mathrm{OH}^{*}+\mathrm{CO}+\mathrm{e}^{-} & \text {(d) } \\ \mathrm{C}_{4} \mathrm{H}_{10} \mathrm{~N}+\mathrm{OH}+\mathrm{CO}^{*}+\mathrm{e}^{-} & \text {(e) }\end{cases}
$$

The realization of channel (a) is confirmed by observation of the spectral lines of the hydrogen atom Balmer series $-\mathrm{H}_{\beta} 486.13 \mathrm{~nm}$ in the wavelength range under investigation (electron energy $30 \mathrm{eV}$ ) [36]. At the electron energy of 50 and $70 \mathrm{eV}$, we can also observe a weak emission of $\mathrm{H}_{\gamma} 434.05$ and $\mathrm{H}_{\delta} 410.17 \mathrm{~nm}$ spectral lines. We note that the same process occurs during the study of acetic acid $\left(\mathrm{CH}_{3} \mathrm{COOH}\right)$ fluorescence [40] and studies of fragmentation, fluorescence, and ionization of formic acid $(\mathrm{CHOOH})[41]$.

Analysis of data available in the literature [41] enables one to identify unambiguously only part of the molecular bands. At an electron energy of $30 \mathrm{eV}$, the main contribution to the emission of a rather wide band at $307 \mathrm{~nm}$ is made by an excited $\mathrm{OH}$ radical (the $\mathrm{A}^{2} \sum^{+}-\mathrm{X}^{2} \mathrm{P}_{\mathrm{i}}(0-0)$ transition, whose radiation wavelengths are split into $306.5+306.8$ and $309.2 \mathrm{~nm}$ ). The emission from this system with vibrational levels 1-1 is manifested at $312.5+314.5 \mathrm{~nm}$, and with vibrational levels $2-2$, it is effectively manifested at 318.5 , $321,323 \mathrm{~nm}$, which is observed in the spectrum. Fragmentation channels (b) and (d) from Eq. (1) are implemented, with the contribution of channel (b) dominating. Comparison with the emission spectrum of the $\mathrm{OH}$ radical $[4,41]$ enables one to identify transitions of the 
same system with vibrational levels $1-0$, which leads to the appearance of bands at 281.2 and $283 \mathrm{~nm}$ and the most intense one at $284 \mathrm{~nm}$. It can also be argued that a band of the $\mathrm{B}^{2} \sum^{+}-\mathrm{A}^{2} \sum^{+}$system at a wavelength of $278.04 \mathrm{~nm}$ is observed as an insignificant maximum. This ratio of the radiation intensities of molecular bands is in agreement with the populations of the excited levels of the radical since the minimum excitation energy of the $\mathrm{A}^{2} \sum^{+}$level is $4.052 \mathrm{eV}$, and for $\mathrm{B}^{2} \sum^{+}$it is $8.65 \mathrm{eV}$.

For the comparing the intensities of the emission bands of the $\mathrm{OH}$ molecule with the vibrational levels 1-0 (283 nm) and 0-0 (307-309 nm) with other excitation methods [33], we calculated the ratio between them, and it is about 0.37 . In our case, at an electron energy of $30 \mathrm{eV}$, this ratio is 0.67 , at $50 \mathrm{eV}$ it is -1.28 , and at $70 \mathrm{eV}$ it is - 1.58 . This means that even at the minimum electron energy, an additional emitter contributes to the emission of the band at $283 \mathrm{~nm}$, and it can be assumed that this is a $\mathrm{CO}^{*}$ molecule (band 3 of the positive group, the $b^{3} \sum^{+}-a^{3} \Pi$ transition with vibrational levels $0-0$ and the wavelength of $283 \mathrm{~nm}$ ). Both $\mathrm{OH}^{*}$ and $\mathrm{CO}^{*}$ excited molecules are fragments formed as a result of an electron impact. An increase in the energy of the bombarding electrons from 30 to $70 \mathrm{eV}$ leads to a redistribution of the fractions of these fragments, which manifests itself as a non-uniform change of corresponding molecular emission intensities.

Thus, it can be concluded that when electrons collide with valine molecules, various channels of its fragmentation are realized with the formation of neutral particles. The most probable of them are the detachment of one of the hydrogen atoms, the $\mathrm{OH}$ radical or the carboxyl group $\mathrm{COOH}$, followed by dissociation into $\mathrm{OH}$ and $\mathrm{CO}$ molecules in the excited state and optical emission. It should be noted that in a study [7] devoted to the formation of various positive ions as a result of electron impact on the valine molecule by mass spectrometry, alternative (apart from the removal of the carboxyl group) valine fragmentation channels are considered. It can be concluded that electron bombardment of valine molecules in the gas phase in the energy range up to $100 \mathrm{eV}$ can form both neutral and positively charged fragments.

\subsection{Electron impact excitation functions}

The technique for measuring the excitation functions (EFs) of molecular emissions of valine under electron impact consisted in fixing the monochromator (Fig. 2) at a certain wavelength and measuring the radiation intensity depending on the energy of the incident electrons. In this case, the most important thing is the calibration of the energy scale of the exciting electron beam and the calibration of the spectral instrument scale. The monochromator wavelength scale was calibrated using a mercury lamp with an accuracy of \pm $0.5 \mathrm{~nm}$. The relative uncertainty of the electron energy scale when measuring the OEF was not worse than \pm $0.1 \mathrm{eV}$. The threshold energy of excitation $\left(E_{\text {ext }}\right)$ of a molecule by electron impact corresponds to the energy of the appearance of a nonzero value of the cross-section in the energy dependence of the EFs. The determination of this energy depends on the following parameters:

The rate of growth of the section at the threshold of the excitation process;

Monoenergeticity $(\Delta E)$ of the exciting electron beam; The accuracy of the calibration of the electron energy scale.

The behavior of the energy dependence of the EFs at the threshold of the collision of electrons with atoms or molecules can be described by the function:

$$
S(E)=k \int_{0}^{\infty} \sigma\left(E-E^{\prime}\right) f\left(E^{\prime}\right) d E^{\prime}
$$

where $\mathrm{k}$ is the detection constant, $E$ is the electron energy provided by the accelerating electrode, $E$ ' is the electron energy in the beam, $\sigma$ is the effective crosssection of the process, $\mathrm{f}\left(E^{\prime}\right)$ is the electron distribution function (Gaussian distribution).

To determine $\mathrm{E}_{\text {ext }}$, one can use the well-known procedure for processing the initial (threshold) part of the energy dependence by the least-squares method using the Marquardt-Levenberg algorithm proposed in Ref. [42]. The threshold section of the experimental excitation function is approximated by the function

$$
F(E)=\left\{\begin{array}{l}
b \quad, E<E_{\text {ext }} \\
b+c \cdot\left(E-E_{\text {ext }}\right)^{n}, E>E_{\text {ext }}
\end{array}\right.
$$

where $b$ is the magnitude of the background signal, $E_{\text {ext }}$ is the energy of the appearance of an excited fragment, $c$ is the coefficient of proportionality, $n$ is the power index in the Wannier threshold law for the energy of the appearance of a fragment ion. The fitting algorithm is implemented using a special computer program that allows one to obtain all four parameters in a large number of iterations, taking into account the value of their standard deviations.

The proposed procedure for determining $E_{\text {ext }}$ was verified for the initial part of the excitation function of the spectral band $\lambda=337.1 \mathrm{~nm}\left(\mathrm{C}^{3} \mathrm{P}_{\mathrm{u}} \rightarrow \mathrm{B}^{3} \Pi_{\mathrm{g}}\right)$ of the nitrogen molecule (see Fig. 5 ). It gave a good agreement between the value of $E_{\text {ext }}$ and the experimental value of the excitation energy. The threshold energy $E_{\text {ext }}$ of an electron, at which one excited fragment is formed due to a breakdown of one bond (see reaction (a) in Eq. (1)), is $E_{\text {ext }}=E_{1}+E_{D}$. In the case of the formation of two excited fragments or three fragments with one excited (see (c) and (d), (e) reactions in Eq. (1)), the threshold energies $E_{\text {ext }}$ are $E_{\text {ext }}=E_{1}+E_{2}+E_{D}$ or $E_{\text {ext }}=E_{1}+E_{D 1}+E_{D 2}$. Here $E_{1}, E_{2}, E_{3}$ are the energies of excitation of fragments, $E_{\mathrm{D}}, E_{\mathrm{D} 1}, E_{\mathrm{D} 2}$ are the energies of bond breaking (dissociation). Hence, we see that the energy $E_{\text {ext }}$ is always greater than the energy (energies) of excitation $E_{\mathrm{k}}$ of the line (lines) by the 


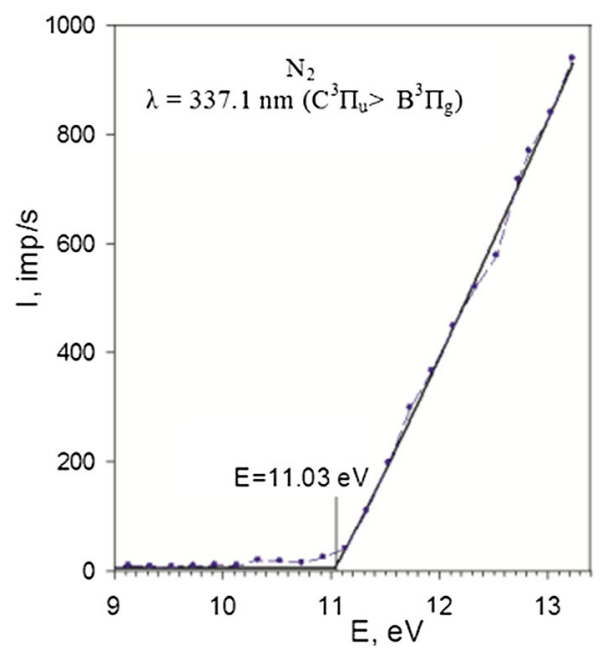

Fig. 5 Threshold part of OEF for $\mathrm{N}_{2}$ molecule: points - experiment, line - approximation by Eq. (3)

amount of energy (energies) of dissociation. The onset of the excitation channel of a new line or another fragmentation channel should affect the OEF behavior.

Using the described method of least squares, the excitation thresholds presented in Table 2 were determined from the excitation functions of the most intense spectral lines. Figure 6 shows the OEF for the most intense molecular emissions. A notable feature of the presented OEFs is a different growth dynamics with an increase in the energy of the exciting electrons and the presence of a number of features.

As can be seen from the figure, the excitation thresholds of these emissions are close to each other, at about $11 \mathrm{eV}$. According to the OEF behavior, they can be attributed to two groups of lines: (1) lines at 284, 335, 353 , and even $386 \mathrm{~nm}$ and (2) lines at 305, $311 \mathrm{~nm}$. In the first group of lines, there is a broad post threshold maximum, which is rather well pronounced for the $353 \mathrm{~nm}$ line with a maximum at $15 \mathrm{eV}$. In the second group, there is a wide $(-15 \mathrm{eV})$ plateau, which for the $305 \mathrm{~nm}$ line begins near $25 \mathrm{eV}$. If we extend the upper part of the curves of the 305 and $311 \mathrm{~nm}$ lines to the $\mathrm{E}$ axis, they will intercept it at about $25 \mathrm{eV}$. Apparently, for both lines, at energies from $15 \mathrm{eV}$, this is the emission of fragments that are separated with some dissociation energies at low electron energies. With increasing the energy of electrons, these fragments emit lines, 305 or $311 \mathrm{~nm}$, but already at high dissociation energies. As a result, the OEF of each of these lines is the sum of their emissions from two identical fragments, which are detached from the valine molecule at low and high electron energies. In the second group, there is a threshold maximum at the beginning, at $-23 \mathrm{eV}$, of the same plateau for the $311 \mathrm{~nm}$ line. Perhaps the maximum is associated with the formation of a negative ion of the valine molecule and its subsequent multi-fragment breakup with excitation of the fragment that gives the $311 \mathrm{~nm}$ line.

The excitation functions of the hydrogen lines $\mathrm{H}_{\beta}$ $486.13 \mathrm{~nm}$ and $\mathrm{H} \gamma \quad 434.05 \mathrm{~nm}$ are somewhat different: the excitation thresholds of these lines are somewhat lower, at about $10.9 \mathrm{eV}$ (see Table 2). Then, with increasing energy of the incident electrons, a gradual increase in the radiation intensity and a number of features are observed similarly to Ref. [29]. Manifestation of these features can be related to a gradual introduction of a-e channels of Eq. (1). Taking into account the low thresholds of OEF for hydrogen lines, the formation of negative ions at low electron energies plays a significant role in the fragmentation of valine.

As shown [23], the formation of negative ions during the interaction of electrons with biological molecules plays an important role in the energy range up to $10 \mathrm{eV}$.

Table 2 Threshold energies and possible identification of the emitters

Wavelength, nm Excitation thresholds, Identification of observed emissions $\mathrm{eV}$ (our data)

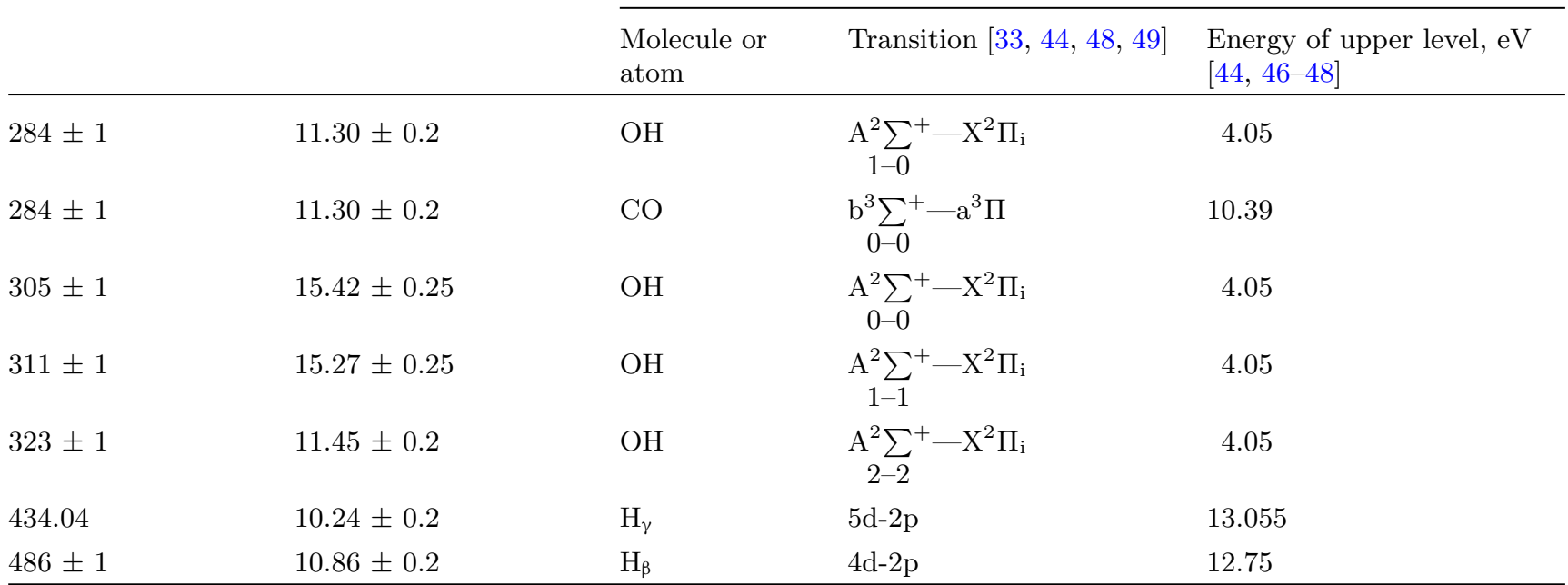



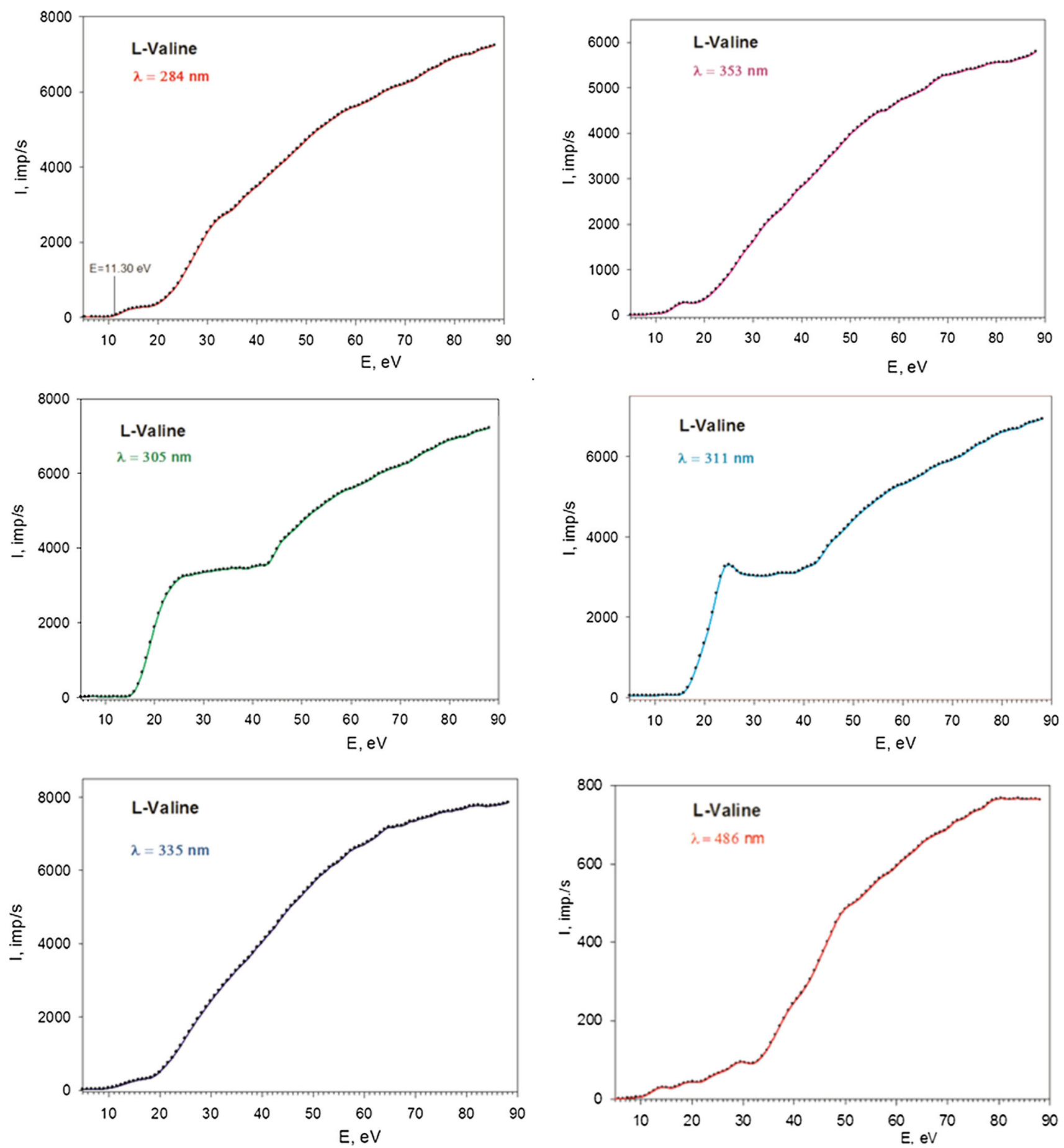

Fig. 6 Optical excitation functions of molecular bands and $H_{\beta}$ spectral line

It should be noted that it was shown [50] that for Lvaline negative ions are formed at low electron energies (5.0-9.0 eV). This leads to the formation of at least 7 fragments of anions: CN, OH, (Val-H), (Val-OH), (Val$\mathrm{COOH}), \mathrm{C}_{3} \mathrm{H}_{6} \mathrm{~N}, \mathrm{C}_{3} \mathrm{H}_{4} \mathrm{O}, \mathrm{COOH}$. The appearance of these negative ions is the result of decay of the $\pi^{*}$ resonance of the $\mathrm{COOH}$ group.
The excitation function of the $\lambda=284 \mathrm{~nm}$ band exhibits a slow growth at the threshold with a small flat maximum at $-17 \mathrm{eV}$, a rather rapid increase from - $19 \mathrm{eV}$, a step at - $32 \mathrm{eV}$, a small break at $58 \mathrm{eV}$, and then an almost linear increase in intensity with increasing electron energy. This is evidence that 


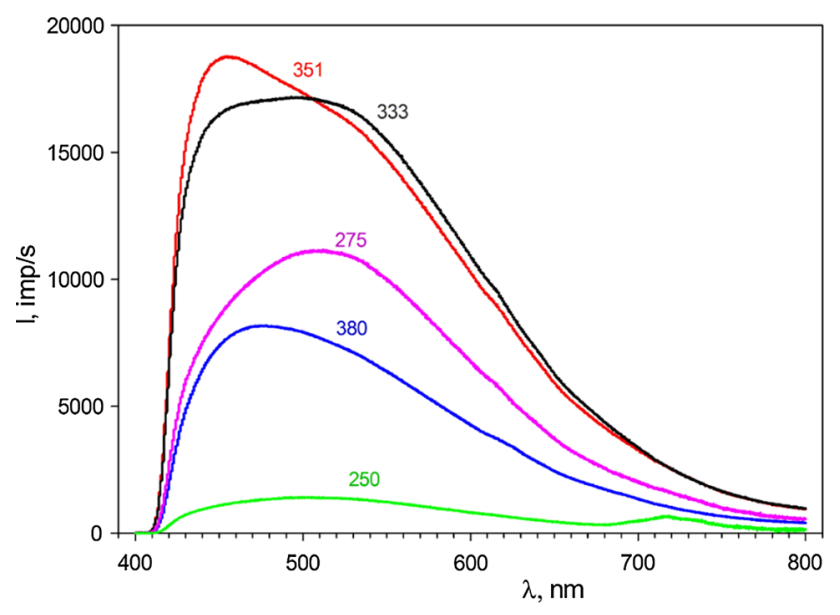

Fig. 7 Luminescence spectra of L-valine measured at 250, $275,333,351$, and $380 \mathrm{~nm}$ excitation wavelengths

there is a competition between (b), (c) and (e) channels of Eq. (1).

The OEFs of the bands with $\lambda=305 \mathrm{~nm}$ and $\lambda$ $=311 \mathrm{~nm}$ behave differently, which in our opinion are emitted by excited $\mathrm{OH}^{*}$ radicals. First, the thresholds for the appearance of these emissions exceed $15 \mathrm{eV}$. With increasing electron energy to $22-23 \mathrm{eV}$, a faster increase in the radiation intensity is observed than for other bands and lines, after which the increase in intensity practically stops. Most likely, we observe the process of direct knocking out of the excited $\mathrm{OH}^{*}$ fragment. The increase in the radiation intensity resumes with an increase in the electron energy above $40 \mathrm{eV}$. At the energy of - $58 \mathrm{eV}$, both OEFs have a kink with a change, decreasing their growth in intensity. In our opinion, this behavior of the OEF of the $\mathrm{OH}^{*}$ radical in the electron energy range of $20 \div 40 \mathrm{eV}$ may be related to the onset of competition between the process of direct knockout of the radical from the valine molecule and a separation of the carboxyl group $\mathrm{COOH}$ followed by its dissociation into $\mathrm{CO}^{*}$ and $\mathrm{OH}^{*}$ fragments. The presence of features in the OEF band $\lambda=284 \mathrm{~nm}$ also testifies in favor of this assumption.

The process of electron bombardment of a valine molecule in the region of low electron energies is as follows. In the energy range up to $7 \mathrm{eV}$, an electron is captured by the valine molecule and the fragmentation begins, as evidenced by the appearance of negative ions in the mass spectra [23]. In the range of electron energies above $8 \mathrm{eV}$, the thresholds for the appearance of positive ions of molecular fragments are observed [21]. And only after an increase in the electron energy above $10.5 \mathrm{eV}$, as shown in this work, neutral excited fragments appear in the form of excited atoms and molecules.

In conclusion, we note that the direct knocking out of a hydrogen atom and other fragmentation mechanisms with the formation of excited neutral molecules may indicate that the valine molecule decays at the moment of interaction with an electron of an appropriate energy. A more detailed analysis of the features we found in the OEF of the investigated emissions requires a special theoretical study as well as elucidation of the important role of the formation of negative valine ions at electron energies below $7 \mathrm{eV}$. A capture of an electron precedes and stimulates fragmentation processes.

\subsection{Photoluminescence spectra}

There are no data in the literature on the luminescence spectra of valine molecules. We measured the luminescence spectrum of powdered L-valine in the spectral range of 400-800 nm. Figure 7 shows a spectrum measured under excitation with five different wavelengths. All spectra have different shapes and intensities of luminescence.

The most intense luminescence is obtained at $\lambda=$ $454 \mathrm{~nm}$ during irradiation of L-valine by

$\lambda=351 \pm 2.5 \mathrm{~nm}$ excitation wavelength. This spectrum also exhibits a second maximum at $\lambda=505 \mathrm{~nm}$. The same shape of the spectrum is observed upon excitation of luminescence by photons with a wavelength $\lambda$ $=333 \pm 2.5 \mathrm{~nm}$. The difference lies in approximately the same luminescence intensity at $\lambda=454 \mathrm{~nm}$ and $\lambda$ $=505 \mathrm{~nm}$.

Noteworthy is the fact that the luminescence spectra measured for $\lambda=275 \pm 2.5 \mathrm{~nm}$ and.

$\lambda=380 \pm 2.5 \mathrm{~nm}$ have different positions of the maxima on 511 and $474 \mathrm{~nm}$, respectively. Moreover, the luminescence intensity in the case of $\lambda=275 \pm 2.5 \mathrm{~nm}$ is $30 \%$ higher. In the spectrum measured at.

$\lambda=250 \pm 2.5 \mathrm{~nm}$, the lowest radiation intensity was observed, and the spectrum itself has a shape with one flat maximum in the long-wavelength region of the spectrum. If the main role in the luminescence spectra of valine is played by molecular excitations, then it can be assumed that the use of exciting photons with energies exceeding the bond energies of most atoms in the valine molecule leads to the lowest quantum yield of luminescence because of the competition between channels of dissociation.

Since the highest luminescence intensity is observed when the excitation wavelengths

$\lambda=351 \pm 2.5 \mathrm{~nm}$ and $\lambda=333 \pm 2.5 \mathrm{~nm}$ are used, it can be assumed that the excitation of $\mathrm{C}-\mathrm{O}$ and $\mathrm{C}-\mathrm{C}$ bonds is dominant (see Table 1). Using the wavelength $\lambda=380 \pm 2.5 \mathrm{~nm}$, effectively excites the $\mathrm{C}-\mathrm{N}$ and $\mathrm{C}-\mathrm{O}$ bonds. An increase in the photon energy for $\lambda=275$ $\pm 2.5 \mathrm{~nm}$ makes it possible to excite the $\mathrm{H}-\mathrm{O}$ and $\mathrm{H}-\mathrm{C}$ bonds. Most likely, this is the reason for the different positions of the maxima in these spectra.

Probably, the observation of a broad emission band with a maximum at $\lambda=474 \mathrm{~nm}$ and $\lambda=505 \mathrm{~nm}$ in the luminescence spectra of $\mathrm{L}$-valine is related to excitation processes in the carboxyl group $\mathrm{COOH}$, which plays a special role in the structure of the valine molecule (Fig. 1). Excitation processes related to this molecule are manifested primarily both when interacting with electrons (which leads to the formation of both excited 
molecules and positively charged ions) and with photons.

\section{Conclusions}

Our experimental studies of the interaction of electrons and photons with the L-valine molecule allow us to draw the following conclusions:

1. When colliding with electrons, a direct detachment of a hydrogen atom and other mechanisms of fragmentation with the formation of excited neutral molecules occur, which indicates the decay of a valine molecule at the moment of interaction with an electron with corresponding energy.

2. Excitation of valine molecules by electrons in the gas phase leads to the appearance in the emission spectrum of both $\mathrm{OH}^{*}, \mathrm{CO}^{*}$ molecular bands and spectral lines of the hydrogen atoms. In the electron energy range $30-70 \mathrm{eV}$, the distribution of radiation intensities between individual bands changes significantly and new molecular emissions appear.

3. A significant part of the processes of fragmentation of L-valine during electron impact occurs with the participation of the carboxyl group $\mathrm{COOH}$, the excitation of which also occurs when interacting with photons.

4. In the luminescence spectrum of L-valine in the wavelength range of 400-800 $\mathrm{nm}$ upon excitation with different wavelength we observed emission bands with maxima at $\lambda=474 \mathrm{~nm}$ and $\lambda=505 \mathrm{~nm}$ which related to excitation in the carboxyl group $\mathrm{COOH}$, which plays the main role in the structure of the valine molecule, especially in the processes of inelastic interactions of photons and electrons with aliphatic amino acids.

5 . Thresholds of the appearance of excited hydrogen atoms and excited molecules are different and its possible influence of the formation of the negative ions states as a whole molecule of valine as well as fragments of its dissociation.

Acknowledgments The authors are deeply grateful to their colleague Yu. M. Azhniuk for useful comments and assistance in preparing the article. The authors are grateful to N. Erdevdi and T.Yu. Popik for help in experiments and E. Yu. Remeta for fruitful theoretical discussions.

\section{Author contribution}

Yu A Bandurin and Sh Molnar contributed to experiments and analysis of their results. A $\mathrm{N}$ Zavilopulo and $\mathrm{O}$ O Shpenik contributed to the analysis of the results. All authors participated in the discussion of results and preparation of the manuscript.

Data Availability Statement This manuscript has associated data in a data repository. [Authors' comment: The data will be deposited in a repository on the web-site of the Institute of Electron Physics (http://iep.org.ua/).]

\section{References}

1. T. D. Mark. "Ionization of Molecules by Electron Impact". In: Electron-Molecule Interactions and Their Applications. 1st ed. L. G. Christophorou, (1984).

2. H. Abdoul-Carime, S. Gohlke, E. Illenberger, Chem. Phys. Let. 402, 497-502 (2005). https://doi.org/10. 1016/j.cplett.2004.12.073

3. M. Dampc, P. Możejko, M. Zubek, Eur. Phys. J. D 72, 216 (2018). https://doi.org/10.1140/epjd/e201890474-2

4. C.G. Pariger, C.M. Helstern, B.S. Jordan et al., Molecules 25, 988 (2020). https://doi.org/10.3390/ molecules25040988

5. I. Fabrikant, S. Eden, N.J. Mason, J. Fedor, Adv. Atom. Mol. Opt. Phys. 66, 546-641 (2017). https://doi.org/10. 1016/bs.aamop.2017.02.002

6. L. Sanche, Radiat. Phys. Chem. 128, 36 (2016). https:// doi.org/10.1016/j.radphyschem.2016.05.008

7. P. Papp, P. Shchukin, J. Kocíšek, Š Matejcík, J. Chem. Phys. 137, 105101 (2012). https://doi.org/10.1063/1. 4749244

8. N.M. Erdevdi, A.I. Bulhakova, O.B. Shpenik, A.N. Zavilopulo, Tech. Phys. Let. 46, 815 (2020). https://doi. org/10.1134/S1063785020080209

9. A.N. Zavilopulo, A.I. Bulhakova, Tech. Phys. Let. 45, 1252 (2019). https://doi.org/10.1134/ S1063785019120290

10. A.N. Zavilopulo, O.B Shpenik., A.N. Mylymko, V.Yu. Shpenik. Int. J. Mass Spectr 441, 1 (2019). https://doi. org/10.1016/j.ijms.2019.03.008

11. O. V. Smirnov, A. A. Basalaev, V. M. Boitsov, S. Yu. Vyaz'min, A. L. Orbeli \& M. V. Dubina, Technical Physics 59, 1698 (2014) https://doi.org/10.1134/ S1063784214110231

12. C. Sonntag, The chemical basis for radiation biology (Taylor \& Francis Press, London, 1987)

13. V.V. Nikiforov, T.G. Suranova, T.Y. Chernobrovkina, Y.D. Yankovskaya, S.V. Burova, Arkh. Vnutr. Med. 10(2), 87 (2020). https://doi.org/10.20514/2226-67042020-10-2-87-93

14. B.K. Romanov, Bezopasn. Risk Farmakoter. 8(1), 3 (2020). https://doi.org/10.30895/2312-7821-2020-8-1$3-8 \mathrm{P}$

15. J.M. van der Burgt, F. Mahon, G. Barrett, M.L. Gradziel, Eur. Phys. J. D 68, 151 (2014). https://doi. org/10.1140/epjd/e2014-40699-0

16. P.J.M. van der Burgt, S. Finnegan, S. Eden, Eur. Phys. J. D 69, 173 (2015). https://doi.org/10.1140/ epjd/e2015-60200-y

17. L. Chen, R. Bredy, J. Bernard, G. Montagne, A.R. Allouche, S.J. Martin, J. Chem. Phys. 135, 114309 (2011). https://doi.org/10.1063/1.3621713

18. B.F. Minaev, M.I. Shafranyosh, YuYu. Svida et al., J. Chem. Phys. 140, 175101 (2014). https://doi.org/10. $1063 / 1.4871881$

19. I.I. Shafranyosh, M.I. Sukhoviya, and M.I. Shafranyosh, J. Phys B: Atomic, Molecular and Optical Physics 39, 4155 (2006). https://doi.org/10.1088/0953-4075/ $39 / 20 / 013$

20. A.N. Zavilopulo, O.B. Shpenik and A.S. Agafonova, J. Phys B: Atomic, Molecular and Optical Physics 42, 
025101 (2009). https://doi.org/10.1088/0953-4075/42/ $2 / 025101$

21. A.N. Zavilopulo, A.I. Bulhakova, S.S. Demes, EYu. Remeta, A.V. Vasiliev, Eur. Phys. J. D 75, 287 (2021). https://doi.org/10.1140/epjd/s10053-021-00294-2

22. A.N. Zavilopulo, S. Demes, E.Yu. Remeta, and A.I. Bulhakova, Ukr. J. Phys. 66(9). 745 (2021). https://doi.org/ 10.15407/ujpe66.9.745

23. S A Pshenichnyuk, N.L. Asfandiarov, A.S. Vorob'ev, Š. Matejčík, Phys. Usp., accepted. https://doi.org/10. 3367/UFNe.2021.09.039054

24. K. Aflatooni, A.M. Scheer, P.D. Burrow, J. Chem. Phys. 125, 054301 (2006). https://doi.org/10.1063/1.2229209

25. K. Aflatooni, G. A. Gallup, and P. D. Burrow, J. Phys. Chem. A 1026205 (71998). https://doi.org/10.1021/ jp980865n

26. I.I. Shafranyosh, M.I. Sukhoviya, M.I. Shafranyosh, L.L. Shimon, Tech. Phys. 53, 1538 (2008). https://doi.org/ 10.1134/S1063784208120025

27. M.A. Rahman, E. Krishnakumar, J. Chem. Phys. 144, 161102 (2016). https://doi.org/10.1063/1.4948412

28. G. Hanel, B. Gstir, S. Denifl, P. Scheier, M. Probst, B. Farizon, M. Farizon, E. Illenberger, T.D. Mark, Phys. Rev. Let. (2003). https://doi.org/10.1103/ PhysRevLett.90.188104

29. O.B. Shpenik, N.M. Erdevdy, V.V. Zvenighorodsky, L.G. Romanova, J. Appl. Spectrosc. 80, 43-46 (2013). https://doi.org/10.1007/s10812-014-9990-x

30. S.G. Stepanian, I.D. Reva, E.D. Radchenko, L.J. Adamowicz, J. Phys. Chem. A 103, 4404-4412 (1999). https://doi.org/10.1021/jp984457v

31. V.S. Shelkovsky, S.G. Stepanian, I.K. Galetich, M.V. Kosevich, L. Adamowicz, Eur. Phys. J. D. 20(3), 421-430 (2002). https://doi.org/10.1140/epjd/e200200144-9

32. S. Dokmaisrijan, V.S. Lee, P. Nimmanpipug, J. Mol. Struct. (Theochem) 953(1-3), 28-38 (2010). https:// doi.org/10.1016/j.theochem.2010.04.033

33. Y. Hu, R. Elliot, J. Bernstein, J. Chem. Phys. 128, 164311 (2008). https://doi.org/10.1063/1.2902980

34. D.H. Mordaunt et al., J. Chem. Phys. 107, 6603 (1997). https://doi.org/10.1063/1.475163

35. V.A. Tverdislov, IOP Journal of Physics: Conference Series 741(1), 012065 (2016). https://doi.org/10.1088/ $1742-6596 / 741 / 1 / 012065$
36. O.B. Shpenik, A.I. Bulhakova, A.N. Zavilopulo, N.M. Erdevdi, Yu.A. Bandurin, Tech. Phys. Let. 47(7), 717 (2021). https://doi.org/10.1134/S1063785021070269

37. B. Shpenik, V.T. Maslyuk, A.N. Zavilopulo et al., J. Phys. B: At. Mol. Opt. Phys. 54, 145201 (2021). https://doi.org/10.1088/1361-6455/ac1360

38. Shimadzu Spectrofluorophotometer RF-6000 Instruction Manual. Shimadzu Corporation. 86. (2015)

39. A. Zavilopulo, Yu. Bandurin, A. Bulhakova. POSMOL 2021. Book of Abstracts 78 (2021)

40. S. Leach, M. Schwell, H.-W. Jochims, H. Baumgärtel, J. Chem. Phys. 321, 171 (2006). https://doi.org/10.1016/ j.chemphys.2005.08.020

41. M. Schwell, F. Dulieu, H.-W. Jochims, J.-H. Fillion, J.-L. Lemaire, H. Baumgärtel, J. Phys. Chem. A 106, 10908 (2002). https://doi.org/10.1021/jp020809t

42. T. Fiegele, G. Hanel, I. Torres et al., J.Phys. B: Atomic Molecular and Optical Physics 33 (20), 4263 (2000). https://doi.org/10.1088/0953-4075/33/20/306

43. P. Papp, J. Urban, Š Matejčík, M. Stano, O. Ingolfsson, J. Chem. Phys. 125, 204301 (2006). https://doi.org/10. $1063 / 1.2400236$

44. K.P. Huber and and G. Herzberg, Van Nostrand Reinhold Company New-York 732 (1979).

45. NIST Atomic Spectra Data base, http://physics. nist.gov

46. R.W.B. Pearse, A.G. Gaydon, The idetification of moleculer spectra (Chapman \& Hall LTD, London, 1965), p. 387

47. J.E. Sansonetti, W.C. Martin and S.L. Young, Handbook of Basic Atomic Spectroscopic Data (2005). https://www.nist.gov/pml/handbook-basicatomicspectroscopic-data

48. A.A. Radzig, B.M. Smirnov, Reference Data on Atoms, Molecules, and Ions (Springer, Berlin, 1986), p. 289

49. Y. Erbil, S. Oztezcan, Life Sci. 78, 376-382 (2006). https://doi.org/10.1016/j.lfs.2005.04.068

50. K. Gaurav, R.K. Goel, M. Shukia et al., Indian J. Med. Paediatr. Oncol. 33, 13-20 (2012). https://doi.org/10. 4103/0971-5851.96962. 